\title{
Synthesis of $N$-Methyl and Azasulfuryl Urotensin-II(4-11) Derivatives
}

\author{
Francesco Merlino ${ }^{1,2}$, Ali M. Yousif ${ }^{1,2}$, Salvatore Di Maro ${ }^{1}$, Stéphane Turcotte $^{2}$, \\ Julien Dufour-Gallant ${ }^{2,3}$, David Chatenet ${ }^{3}$, Paolo Santicioli ${ }^{4}$, Ettore Novellino ${ }^{1}$, \\ Paolo Grieco ${ }^{1}$, and William D. Lubell ${ }^{2}$ \\ ${ }^{1}$ Department of Pharmacy, University of Naples "Federico II", Naples, 80131, Italy; ${ }^{2}$ Département de Chimie, \\ Université de Montréal, Montréal, H3C 3J7, Canada; ${ }^{3}$ INRS-Institut Armand-Frappier, Institut \\ National de la Recherché scientifique, Université du Québec, Laval, H7V 1B7, Canada; \\ ${ }^{4}$ Department of Pharmacology, Menarini Ricerche, Florence, I-50131, Italy
}

\section{Introduction}

Human urotensin-II ( $h \mathrm{U}-\mathrm{II})$ is a cyclic peptide that is able to regulate cardiovascular homeostasis [1]. The shortest active sequence required for biological activity of $h \mathrm{U}-\mathrm{II}$ contains residues $4-11$ in a cycle featuring a disulfide bond between $\mathrm{Cys}^{5}-\mathrm{Cys}^{10}$, Asp$c$ [Cys-Phe-Trp-Lys-Tyr-Cys]-Val-OH (Figure 1), and is suggested to adopt a $\beta$-hairpin conformation of prime relevance for interaction with the urotensin-II receptor (UT) [2]. Employing the minimal active sequence, we have developed synthetic strategies to prepare new derivatives in which $N$-methylated (1) and azasulfuryl (2) residues have been inserted into the cyclic peptide core region (Figure 1). Mono and multiple $N$-methylation of the amide bonds and insertion of $N$-aminosulfamide residues into $h \mathrm{U}-\mathrm{II}_{(4-11)}$ have been performed to survey their influences on intra- and inter-molecular hydrogen bonds, geometry and interactions with UT.
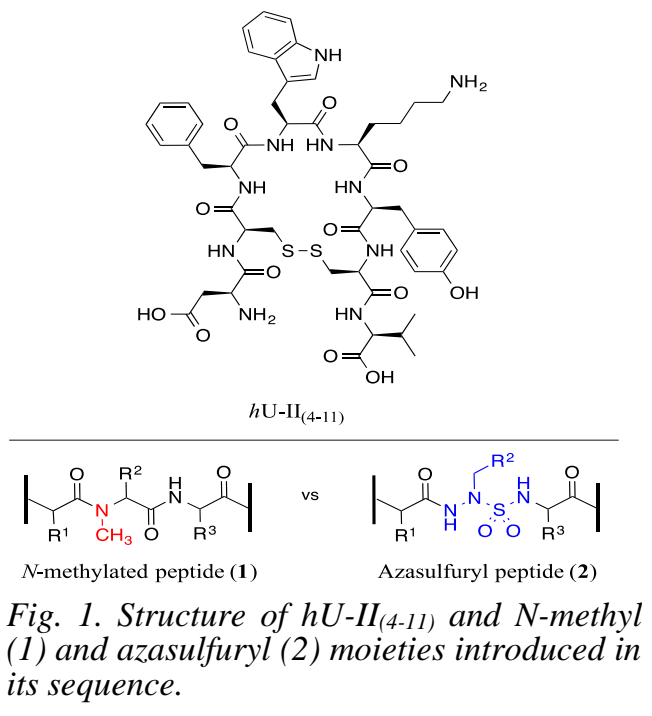

Fig. 1. Structure of $h U-I I_{(4-11)}$ and $N$-methyl (1) and azasulfuryl (2) moieties introduced in its sequence.

\section{Results and Discussion}

A solid-phase procedure was used to install $N$-methyl groups on amides at residues 5-10 consisting of three fundamental steps: i) amine protection with the $o$-nitrobenzenesulfonyl group (o-NBS), ii) amine alkylation with methylsulfate and 1,8-diazabicycloundec-7-ene (DBU) as base, and iii) selective removal of the $o$-NBS group on solid support (Scheme 1) [3].

$N$-Aminosulfamides are peptidomimetics in which the $\mathrm{C}_{\alpha} \mathrm{H}$ and the carbonyl of an amino amide residue are respectively replaced by a nitrogen atom and a sulfuryl group. Azasulfurylphenylalanine (AsF) analogue 8 was synthesized by an approach consisting of two phases: i) the synthesis of the AsF tripeptide building block 5 by solution phase chemistry with protected functional groups compatible
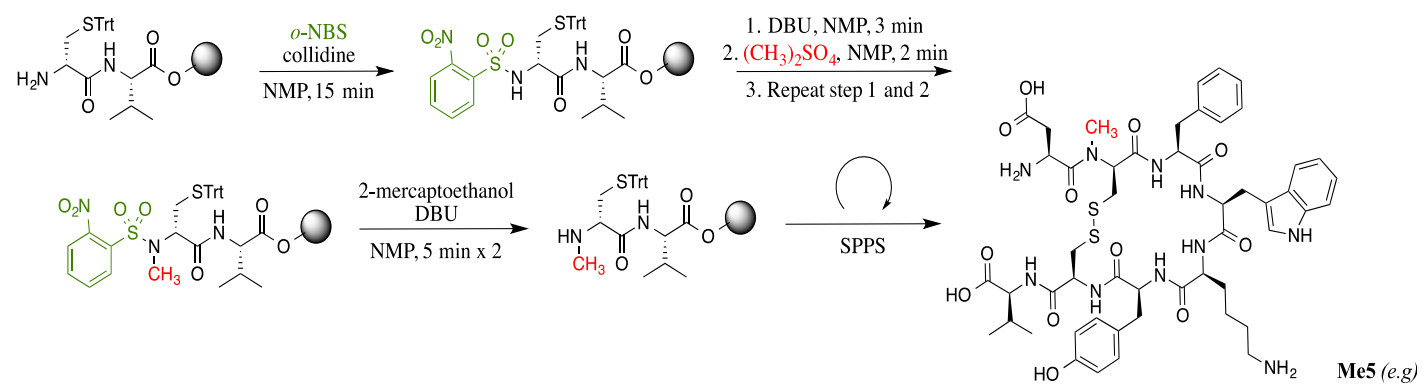

Scheme 1. Three-step procedure for the synthesis of N-methylated U-II analogues is illustrated for the preparation of $\left[\mathrm{N}-(\mathrm{Me}) \mathrm{Cys}^{5}\right] \mathrm{hU}-\mathrm{II}_{(4-11)}(\mathrm{Me} 5)$. 
with solid phase peptide synthesis (Scheme 2a); ii) incorporation of AsF tripeptide 6 into the peptide sequence on solid support using a Fmoc/tBu orthogonal protection strategy (Scheme $2 \mathrm{~b}$ ). In $\left[\mathrm{AsF} \mathrm{F}^{7} h \mathrm{U}-\right.$ $\mathrm{II}_{(4-11)}(\mathbf{8})$, the $\operatorname{Trp}^{7}$ residue was replaced by azasulfurylphenylalanine with the phenyl group serving as a mimic of the indole moiety. Initially, the AsF tripeptide building block $\mathbf{5}$ was synthesized using a solution-phase approach featuring chemoselective alkylation of azasulfurylglycine (AsG) $\mathbf{4}$ with benzyl bromide and tert-butylimino-tri(pyrrolidino)phosphorane (BTPP) as base [4].
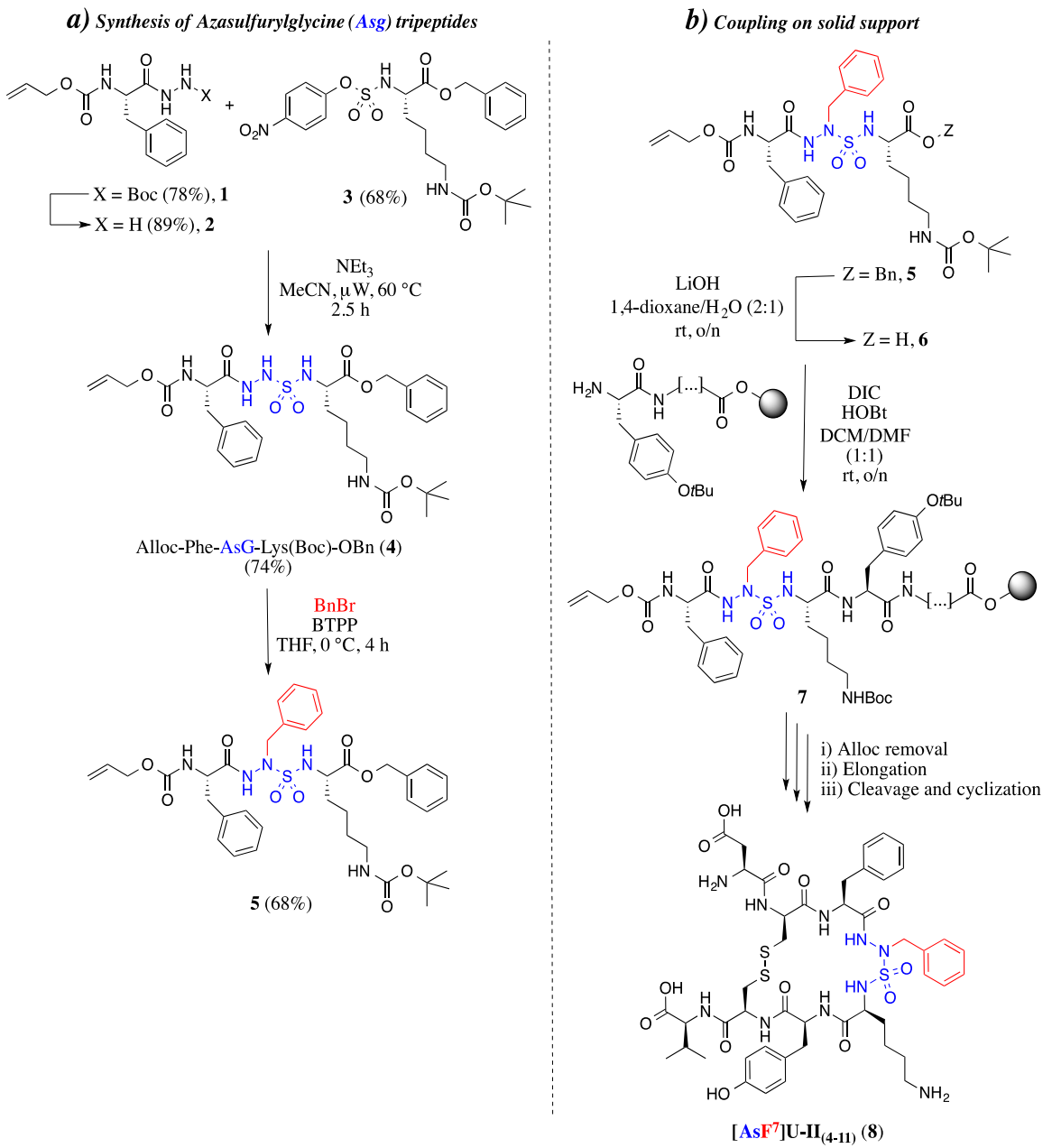

Scheme 2. Synthesis of $\left[A s F^{7}\right] h U-I I_{(4-11)}(8):$ a) preparation of azasulfurylphenylalainine (AsF) tripeptide 5 ; $b)$ introduction of AsF tripeptide into $\left[A s F^{7}\right] U-I I_{(4-11)}(8)$.

With AsF tripeptide building block 6 in hand, solid phase chemistry was performed on Rink amide resin. Azasulfuryl tripeptide 6 was coupled to the $\mathrm{Tyr}^{9}$ residue using di-iso-propylcarbodiimide and hydroxybenzotriazole. Subsequently, the $N$-terminal Alloc group was removed using palladium catalysis and the sequence was elongated by conventional solid phase peptide synthesis (SPPS) [5]. Cleavage and disulfide bond formation were accomplished in a one-pot reaction using a cocktail of $10 \%$ DMSO in TFA in the presence of anisole as scavenger to afford azasulfuryl peptide $\mathbf{8}$, which was purified by HPLC on a C18 bonded silica column and characterized by LCMS and HRMS analysis $\left[\mathrm{t}_{\mathrm{R}}=14.18 \mathrm{~min}, 20\right.$ to $80 \%$ methanol $(0.1 \%$ formic acid $)$ in water $(0.1 \%$ formic acid $)$ over $15 \mathrm{~min}$; 
Table 1. Biological data for mono $N$-methylated $h U-$ II $_{(4-11)}$ analogues.

\begin{tabular}{cccc}
\hline \multirow{2}{*}{ Peptide } & Sequence & \multicolumn{2}{c}{ Biological data } \\
& & $p E C_{50}{ }^{a}$ & $p K_{D} / p K_{i}{ }^{b}$ \\
\hline $\boldsymbol{h}$ U-II $(\mathbf{4}-\mathbf{1 1})$ & Asp-c[Cys-Phe-Trp-Lys-Tyr-Cys]-Val-OH & $8.20 \pm 0.01$ & $8.34 \pm 0.04$ \\
Me5 & Asp-c[(NMe)Cys-Phe-Trp-Lys-Tyr-Cys]-Val-OH & $8.26 \pm 0.03$ & $8.53 \pm 0.08$ \\
Me6 & Asp-c[Cys-(NMe)Phe-Trp-Lys-Tyr-Cys]-Val-OH & $6.36 \pm 0.04$ & $6.64 \pm 0.10$ \\
Me7 & Asp-c[Cys-Phe-(NMe)Trp-Lys-Tyr-Cys]-Val-OH & $\mathbf{8 . 5 0} \pm \mathbf{0 . 0 5}$ & $\mathbf{8 . 7 6} \pm \mathbf{0 . 0 7}$ \\
Me8 & Asp-c[Cys-Phe-Trp-(NMe)Lys-Tyr-Cys]-Val-OH & $4.91 \pm 0.18$ & $=5$ \\
Me9 & Asp-c[Cys-Phe-Trp-Lys-(NMe)Tyr-Cys]-Val-OH & $5.25 \pm 0.07$ & $=5$ \\
Me10 & Asp-c[Cys-Phe-Trp-Lys-Tyr-(NMe)Cys]-Val-OH & $8.31 \pm 0.08$ & $8.45 \pm 0.05$ \\
\hline
\end{tabular}

a functional activity was tested on the rat thoracic aorta; ${ }^{b}$ binding affinity was evaluated on UT receptors expressed on $\mathrm{CHO}$ cell line.

calculated mass: 1059.3733, found: 1059.3706]. Similar procedures are being pursued to broaden the library of azasulfuryl peptide mimics of the $h \mathrm{U}-\mathrm{II}_{(4-11)}$ sequence.

Biological data for the $N$-methyl $\mathrm{U}-\mathrm{II}_{(4-11)}$ analogs was acquired through experiments performed on $\mathrm{CHO}$ cell lines expressing the human UT receptor for binding affinity, and on rat thoracic aorta for functional activity, according to experimental procedures previously described [6]. In agreement with the hairpin conformation, $N$-methylation of amide bonds of $\mathrm{U}_{-\mathrm{II}_{(4-1)}}$ at $\mathrm{Phe}^{6}$ and $\mathrm{Tyr}^{9}$ decreased binding affinity and consequently reduced vasoconstriction in the rat thoracic aorta assay (Table 1).

In contrast, $N$-methylation of $\operatorname{Trp}^{7}$ (e.g., Me7) improved affinity relative to the parent peptide. On the other hand, $N$-methylation of the amide bonds at position 8 , which is normally occupied by Lys, decreased affinity, and reduced vasoconstriction in the rat thoracic aorta assay. $N$-Methylation of the Cys residues at positions 5 and 10 gave more active analogs (e.g., Me5 and Me10) without significant change in binding affinity. Preliminary data inspired investigation of a set of di-methylated analogs, which were synthesized by a similar process and are presently being investigated for biological activity. The influence of $N$-methylation on conformation is also being analyzed by NMR spectroscopy.

Other azasulfuryl amino acids are being inserted into the cyclic portion of the $h \mathrm{U}-\mathrm{II}_{(4-11)}$ sequence by replacement of the $\operatorname{Trp}^{7}$ and Lys ${ }^{8}$ amino acid residues. Their synthesis and activity will be reported in due time.

Information from $N$-methylation and $N$-aminosulfamide studies is designed to provide a detailed view of the influences of hydrogen-bonding in the $h \mathrm{U}-\mathrm{II}_{(4-11)}$ sequence. In preliminary results, removal of the N-H group of $\operatorname{Trp}^{7}$ by methylation enhanced interaction with the UT receptor. Further investigation of the urotensin analogues is ongoing and their impact on structure-activity relationships of the urotensinergic system will be reported in due time.

\section{Acknowledgments}

This work was supported in part by the Italian Ministry of Education, University and Research (PRIN2010MCLBCZ_002), a grant from "Regione Campania" - Laboratori Pubblici progetto "Hauteville", and the Natural Sciences and Engineering Research Council of Canada (NSERC).

\section{References}

1. Vaudry, H., et al. Pharmacol. Rev. 67, 214-258 (2015), http://dx.doi.org/10.1124/pr.114.009480

2. Carotenuto, A., et al. J. Pept. Sci. 19, 293-300 (2013), http://dx.doi.org/10.1002/psc.2498

3. Miller, S., Scanlan, T.S. J. Am. Chem. Soc. 119, 2301-2302 (1997), http://dx.doi.org/10.1021/ja9635443

4. Turcotte, S., et al. Org. Lett. 14, 1318-1321 (2012), http://dx.doi.org/10.1021/ol3001987

5. Lubell, W.D., et al. (2005) "Peptides" Science of Synthesis 21.11, Chemistry of Amides. Thieme, Stuttgart, 713-809.

6. Carotenuto, A., et al. J. Med. Chem. 57, 5965-5974 (2014), http://dx.doi.org/10.1021/jm500218x 